LB3.258 RATES OF PELVIC INFLAMMATORY DISEASE AND ECTOPIC PREGNANCY ARE NO LONGER DECLINING: AN ECOLOGICAL ANALYSIS OF AUSTRALIAN HOSPITAL ADMISSIONS AND EMERGENCY PRESENTATION DATA, 2009-2014

\begin{abstract}
${ }^{1}$ Jane L Goller, ${ }^{1}$ Alysha De Livera, ${ }^{2}$ Rebecca J Guy, ${ }^{3}$ Nicola Low, ${ }^{2}$ Basil Donovan, ${ }^{2}$ Mathew Law, ${ }^{2} J o h n$ Kaldor, ${ }^{4}$ Christopher K Fairley, ${ }^{1}$ Jane S Hocking. ${ }^{1}$ Melbourne School of Population And Global Health, University of Melbourne, Parkville, Australia; ${ }^{2}$ Kirby Institute, Unsw, Sydney, Australia; ${ }^{3}$ University of Bern, Bern, Switzerland; ${ }^{4}$ Central Clinical School, Monash University And Melbourne Sexual Health Centre, Melbourne, Australia
\end{abstract}

10.1136/sextrans-2017-053264.493

Introduction Pelvic inflammatory disease (PID) and ectopic pregnancy (EP) among women are important sequelae of sexually transmissible infections (STIs). We assessed recent trends in these STI-related morbidities in three Australian states (Victoria, New South Wales, Queensland).

Methods Hospital admission and emergency presentation PID and EP rates among women 15-44 years were extracted and analysed by residential postcode for 2009-2014 using population and live birth denominators where relevant. Final data were available in 2017. Zero Inflated Poisson (ZIP) models were used to assess variation in rates by year, age, socio-economic disadvantage and area of residence. A sub-analysis of acute and/or STI-confirmed PID admissions was undertaken.

Results Admission and emergency presentation rates respectively per 100000 women in 2014 were: i) 63.3 (95\% CI: 60.8-65.9) and 97.0 (95\%CI: 93.9-100.2) for PID; and ii) 107.8 (95\%CI: 104.5-111.2) and 96.7 (95\%CI: 93.6-99.9) for EP. Of all emergency cases, 68\% of PID and 22\% of EP were managed without admission. PID admission rates did not change by year, but acute/STI-confirmed PID admissions increased by $40 \%$ between 2009 and 2014 (Incidence rate ratio [IRR]: 1.4; 95\% CI: 1.2-1.7). Emergency PID rates increased by $30 \%$ between 2009 and 2014 (IRR: 1.3; 95\% CI: 1.2-1.5). PID admission and emergency rates were highest among women 15-24 years. Population based EP rates increased by $10 \%$ in emergency between 2009 and 2014 (IRR: 1.1; 95\% CI: 1.1-1.2). EP rates per 1000 live births increased by $8 \%$ (IRR: 1.08 ; 95\% CI: 1.06-1.11) for admissions and 27\% (IRR: 1.27; 95\% CI: 1.21-1.33) for emergency between 2009 and 2014. Increasing disadvantage and remoteness of area tended to be associated with higher PID and EP rates.

Conclusion These data show that, for the first time in two decades, STI-related sequelae diagnoses at Australian hospitals are increasing.

\section{LB3.259 E-STI TESTING AND RESULTS SERVICE: A SINGLE BLIND RANDOMISED CONTROLLED TRIAL}

${ }^{1}$ Emma Wilson, Dr. ${ }^{1}$ Caroline Free, ${ }^{2}$ Tim P Morris, ${ }^{3}$ Jonathan Syred, Dr. ${ }^{4}$ Anatole S MenonJohansson, Dr. 'Melissa J Palmer, ${ }^{3}$ Sharmani Barnard, ${ }^{1} E m m a$ Rezel, Dr. ${ }^{3}$ Paula Baraitser. ${ }^{1}$ London School of Hygiene And Tropical Medicine, London, UK; ${ }^{2}$ Mrc Clinical Trials Unit At University College London, London, UK; ${ }^{3}$ Kings College London, London, UK; ${ }^{4} G u y$ 's And St. Thomas' Nhs Foundation Trust, London, UK

\section{$10.1136 /$ sextrans-2017-053264.494}

Introduction STI self-sampling accessed via the internet (e-STI testing) is recommended to expand access to services. There is limited evidence on its effectiveness. This is the first RCT to evaluate an e-STI testing service for chlamydia, gonorrhoea, HIV and syphilis.

Methods Single-blind RCT with allocation concealment.

Eligibility: aged 16-30, resident in 2 boroughs of London, having at least one sexual partner in the last 12 months, willing to take an STI test.

Participants were randomly allocated to (1) an e-STI testing service or (2) to a website with signposting to local sexual health clinics.

Primary outcomes were: 1) diagnosis of any STI 2) completion of any STI test

All analyses were intention-to-treat. We used multivariate imputation using chained equations (MICE) for the primary analyses. We explored heterogeneity by age, gender, ethnicity, deprivation, number of sexual partners in the last 12 months, and sexuality.

Results 2072 participants were randomised. The response rate was $84 \%$. At 6 weeks, $50.0 \%$ of the intervention group completed an STI test compared to $26.6 \%$ in the control group (RR 1.87, 95\% confidence interval 1.63 to $2.15, \mathrm{p}<0.0001$ ). $2.8 \%$ of the intervention $\mathrm{v} 1.4 \%$ in the control were diagnosed with an STI (RR 2.10, 95\% confidence interval 0.94 to $4.70, p=0.079)$. The effect on cases treated was $1.1 \%$ in the intervention $v 0.7 \%$ in the control (RR 1.72, 95\% confidence interval 0.71 to $4.16, \mathrm{p}=0.231$ ).

No heterogeneity was observed in the pre-specified sub group analyses.

Time-to-test was lower in the intervention arm compared to the control arm (28.8 days vs 36.5 days; $p<0.0001)$. No differences were observed for time-to- treatment (83.2 days vs 83.5 days; $\mathrm{p}=0.51$ ).

Data cleaning and data collection were still underway late 2016.

Conclusion e-STI testing increased testing uptake and may yield a small increase in STI diagnoses. Service innovations may be needed so that gains in testing and diagnoses translate into similar gains in cases treated. e-STI testing could be a valuable option in high prevalence contexts where expanding access is priority.

\section{LB3.260 MULTIDRUG RESISTANT MYCOPLASMA GENITALIUM IN HIV-INFECTED MEN WHO HAVE SEX WITH MEN (MSM) IN THE UNITED STATES}

Li Xiao; William M Geisler, Ken B Waites, Kristal J Aaron, Jodie A Dionne-Odom. University of Alabama at Birmingham, Birmingham, USA

\subsection{6/sextrans-2017-053264.495}

Introduction Mycoplasma genitalium (MG) is a common cause of urethritis in men. In the U.S., CDC recommends treatment for MG with azithromycin or moxifloxacin, but treatment success is threatened by increasing resistance to both therapies. Rectal MG infection has been described among MSM, but little is known about rectal MG prevalence and frequency of antibiotic-resistant MG in HIV-infected MSM.

Methods We retrospectively evaluated the prevalence of MG infection and antibiotic-resistant MG in 158 HIV-infected MSM who self-collected rectal and urine samples for a study at an HIV clinic in Birmingham, Alabama in 2014-2016. Eligibility criteria included receptive anal intercourse in the past 30 days and no recent antibiotic exposure (except trimethoprim-sulfamethoxazole). A real-time PCR assay was used to 
detect MG and macrolide resistance by targeting the 23S rRNA gene. Nested PCRs were used to detect mutations in quinolone resistant determination regions in $g y r A, \operatorname{gyr} B$, parC and parE genes.

Results MG infection was detected in 27 MSM (17.1\%); 18 $(11.4 \%)$ at the genital site and $9(5.7 \%)$ at the rectal site. The bacterial load ranged from 2-32,700 genome copies/ $\mu$ l. Macrolide resistant MG was detected in 19 men (70.4\%), featuring typical 23S rRNA mutations (A2071G or A2072G transitions). One subject with MG had novel gene mutations (G1972T and G2038T) with unknown function. Eight (29.6\%) had fluoroquinolone-resistant $\mathrm{MG}$ harbouring parC mutations that cause changes in amino acid position 83 (S83I or S83R); 4 of them had an additional P62S mutation in parC and 1 had a F475S mutation in gyrB. Five men (18.5\%) had MG with dual macrolide and fluoroquinolone resistance. The prevalence of resistance was similar at rectal and genital sites.

Conclusion This is the first U.S. study to document a high frequency of macrolide and fluoroquinolone-resistant $\mathrm{MG}$ in HIV-infected MSM at rectal and genital sites. If these resistance mutations are associated with clinical treatment failure, more effective options to treat MG are needed.

\section{LB3.261 TRENDS IN CONDOM USE AND SEXUAL POSITIONING AMONG MEN-WHO-HAVE-SEX-WITH-MEN IN THE ERA OF HIV PRE-EXPOSURE PROPHYLAXIS, AND RISK FOR DIAGNOSES OF INCIDENT HIV AND OTHER SEXUALLY TRANSMITTED INFECTIONS - NEW YORK CITY, 2011- 2015}

Preeti Pathela, Kelly Jamison, Sarah Braunstein, Julia Schillinger. New York City Department of Health And Mental Hygiene, Queens, USA

\subsection{6/sextrans-2017-053264.496}

Introduction Men-who-have- sex-with-men (MSM) may modify sexual practices to reduce HIV and sexually transmitted infection (STI) risk. HIV pre-exposure prophylaxis (PrEP) may impact risk behaviour and STI acquisition.

Methods We matched HIV-negative MSM attending New York City (NYC) sexual health clinics during 2011-2015 to the NYC HIV registry in $01 / 2017$. We used visit-level data to assess trends in condom use during anal sex (consistent, inconsistent, no use; referent period $=3$ months), overall and by sexual positioning behaviour. We examined associations between condomless (inconsistent/no use) insertive, condomless receptive, and condomless versatile sex and incident HIV or STI (chlamydia/gonorrhoea/early syphilis). We used regression with generalised estimating equations (referent group $=$ anal sex with condoms), controlling for demographics, partner number, and STI history.

Results The proportion of visits with reported consistent condom use decreased from 2011-2015 (39\% to $31 \% \%$, $\mathrm{p}<0.001)$; inconsistent use increased $(48 \%$ to $55 \% \%)$, and no condom use was stable (13\%-14\%). There were significant declines in consistent condom use across all positioning categories. From all visits, MSM reported positioning as: 19\% condomless insertive, 9\% condomless receptive, 37\% condomless versatile, 35\% sex with condoms. For 25,216 STI testing visits that yielded 7438 diagnoses, all condomless-positioning categories were associated with incident STI; highest risk was with condomless insertive sex (aOR 1.8, 95\% CI 1.6-1.9). For MSM tested for HIV at 9744 visits, condomless receptive (aOR 2.8; 95\% CI 1.9-4.1) and condomless versatile sex (aOR 2.2; 95\% CI 1.6-2.8) were associated with incident HIV. Black MSM ( $25 \%$ of MSM) had the highest risk for STI and HIV (41\% of 368 new HIV diagnoses).

Conclusion We documented increases in condomless sex among a sentinel high risk group prior to and during the PrEP era. Insertive sex, perhaps perceived as a safer strategy, was associated with substantial STI risk when condoms were not consistently used. Assuring PrEP access for black MSM is critical.

\section{LB3.262 LATE POSTNATAL HIV MOTHER TO CHILD TRANSMISSION THROUGH BREASTFEEDING: ANALYSIS OF INFANT CASES OF PREVIOUSLY SERONEGATIVE MOTHERS INFECTED DURING LACTATION}

Ana Carolina Barcellos, Nathalia Zorze Rossetto, Cristina de Oliveira Rodrigues. Universidade Federal do Paraná, Curitiba - PR, Brazil

\subsection{6/sextrans-2017-053264.497}

Introduction Among children, the main postnatal HIV category of exposure is breastfeeding. When acute maternal infection occurs during lactation, the high maternal viral rate results in a higher risk of infant infection. There are few researches showing the impact of HIV infection on children of previously seronegative mothers, who acquire the HIV virus during breastfeeding. To elucidate the importance of this category of exposure, this study aims to evaluate HIV infection in infants assisted by a Paediatric Infectology Service in Brazil, emphasising the cases where vertical transmission occurred lately through breastfeeding.

Methods Transversal, analytical and descriptive study, with quantitative and qualitative approach analysing HIV infected patients from 0 to 16 years old. Data was collected during 2016, from charts of children assisted between 2010 and 2015. After previous selection, breastfed children of mothers who had negative HIV testing during pregnancy and/or at birth, had charts analysed in detail.

Results From a total of 122 cases, 95\% were mother-to-childtransmissions. Between these cases, 11 were considered possible/confirmed late postnatal transmission through breastfeeding. This group characterisation showed that at diagnosis, $72,7 \%$ presented significant and/or recurrent symptoms of HIV infection. In $45,4 \%$, mother and children were diagnosed at the same time and $72,7 \%$ of mothers were sexually infected.

Conclusion There was a significant prevalence of late postnatal transmission through breastfeeding in our sample. Differently from resource limited settings, most countries avoid breastfeeding by HIV infected mothers. Considering cases where women were infected only during lactation, our study highlights a gap in prevention of vertical transmission of HIV. The severity of infant symptoms, the moment of diagnosis and mother's category of exposure confirm the importance of preventive measures and scientific improvement to reduce postnatal HIV transmission. 